\title{
Revisiting scaling laws in river basins: New considerations across hillslope and fluvial regimes
}

\author{
Chandana Gangodagamage, ${ }^{1,2}$ Patrick Belmont, ${ }^{1,3}$ and Efi Foufoula-Georgiou ${ }^{1}$ \\ Received 2 March 2010; revised 4 April 2011; accepted 18 April 2011; published 7 July 2011.
}

[1] Increasing availability of high-resolution $(1 \mathrm{~m})$ topography data and enhanced computational processing power present new opportunities to study landscape organization at a detail not possible before. Here we propose the use of "directed distance from the divide" as the scale parameter (instead of Horton's stream order or upstream contributing area) for performing detailed probabilistic analysis of landscapes over a broad range of scales. This scale parameter offers several advantages for applications in hydrology, geomorphology, and ecology in that it can be directly related to length-scale dependent processes, it can be applied seamlessly across the hillslope and fluvial regimes, and it is a continuous parameter allowing accurate statistical characterization (higher-order statistical moments) across scales. Application of this scaling formalism to three basins in California demonstrates the emergence of three distinct geomorphic regimes of divergent, highly convergent, and moderately convergent fluvial pathways, with notable differences in their scaling relationships and in the variability, or spatial heterogeneity, of topographic attributes in each regime. We show that topographic attributes, such as slopes and curvatures, conditional on directed distance from the divide exhibit less variability than those same attributes conditional on upstream contributing area, thus affording a sharper identification of regime transitions and increased accuracy in the scaling analysis.

Citation: Gangodagamage, C., P. Belmont, and E. Foufoula-Georgiou (2011), Revisiting scaling laws in river basins: New considerations across hillslope and fluvial regimes, Water Resour. Res., 47, W07508, doi:10.1029/2010WR009252.

\section{Introduction}

[2] The organization of landscapes and stream networks influences many processes in hydrology, geomorphology, and terrestrial/aquatic ecology. Many different approaches have been developed to quantify the morphology and hierarchical organizational structure of drainage basins and understand how the physical attributes of a drainage basin change as a function of scale [Horton, 1945; Hack, 1957; Shreve, 1966; Shreve, 1967; Tarboton, 1992; RodríguezIturbe et al., 1992; Ijjasz-Vasquez and Bras, 1995; Tarboton, 1996; Rigon et al., 1996; Maritan et al., 1996; Banavar et al., 1997; Rodríguez-Iturbe and Rinaldo, 1997; Rigon et al., 1998; Rinaldo and Rodriguez-Iturbe, 1998; Banavar et al., 2001; Dodds and Rothman, 2000a; Tucker and Whipple, 2002; Veitzer et al., 2003; Mantilla et al., 2006; Tarolli and Dalla Fontana, 2009]. Selection of the proper scale parameter is critical for understanding the variability and organization of basin morphology, identifying tran-

\footnotetext{
${ }^{1}$ Department of Civil Engineering, National Center for EarthSurface Dynamics, and St. Anthony Falls Laboratory, University of Minnesota, Twin Cities, Minneapolis, Minnesota, USA.

${ }^{2}$ Now at Earth and Environment Sciences Division and Space and Remote Sensing Division, Los Alamos National Laboratory, Los Alamos, New Mexico, USA.

${ }^{3}$ Department of Watershed Sciences, Utah State University, Logan, Utah, USA.
}

Copyright 2011 by the American Geophysical Union. 0043-1397/11/2010WR009252 sitions in geomorphic processes shaping the landscape, and ultimately for validating landscape evolution models across a range of scales.

[3] The organizational structure of river networks has been studied for several decades. Horton introduced the notion of "stream order" $(\omega)$, which organizes the fluvial network into stream segments starting from the point of channel initiation to the stream outlet [Horton, 1945]. This ordering system was later modified by Strahler to better represent the hierarchical structure of a branching network (Horton's original system gave the highest rank to the entire mainstream, from outlet to headwaters, whereas Strahler only gives the highest rank to the stream segment of the mainstream below the confluence of the two next lower order streams) and is known as Horton-Strahler (HS) ordering [Strahler, 1952, 1957].

[4] Horton's laws refer to the scaling relationships of the number of streams, average length of stream segments, and average upstream contributing areas parameterized in terms of the scale parameter $\omega$, where $\omega$ is equal to or less than the maximum basin order $(\Omega)$ :

$$
\begin{aligned}
N(\omega) & \propto R_{B}^{\Omega-\omega} & R_{B}=\frac{N(\omega)}{N(\omega+1)} \\
\left\langle\ell_{S}(\omega)\right\rangle & \propto R_{L s}^{\omega-1} & R_{L s}=\frac{\left\langle\ell_{S}(\omega)\right\rangle}{\left\langle\ell_{S}(\omega-1)\right\rangle} \\
\langle A(\omega)\rangle & \propto R_{A}^{\omega-1} & R_{A}=\frac{\langle A(\omega)\rangle}{\langle A(\omega-1)\rangle}
\end{aligned}
$$


where $N(\omega)$ is the number of order $\omega$ streams; $\left\langle\ell_{s}(\omega\rangle\right.$ is the average segment length of order $\omega ;\langle A(\omega)\rangle$ is the average contributing area of order $\omega$ streams; and $R_{B}, R_{L s}$, and $R_{A}$ are the (scale-independent) bifurcation ratio, length ratio, and area ratio, respectively (collectively called Horton's ratios). For natural channel networks, $R_{B}$ typically ranges between 3 and $5, R_{L s}$ between 2 and 3 , and $R_{A}$ between 3 and 6 [e.g., Valdés et al., 1979; Abrahams, 1984; Dodds and Rothman, 2000b].

[5] The HS ordering system has been adopted as the most common approach for quantifying scaling and for describing the hierarchical structure of river networks. One of the obvious limitations however of using $\omega$ as the scale parameter is the a priori assumption that channel initiation points can be accurately identified. Despite considerable research on this topic [e.g., Montgomery and Dietrich, 1988; Tarboton et al., 1991; Dietrich and Dunne, 1993; Montgomery and Foufoula-Georgiou, 1993] (see also the recent developments by Lashermes et al. [2007], Passalacqua et al. [2010], and Pirotti and Tarolli [2010] for high-resolution DEMs), channel source identification from DEMs remains a challenge and often requires calibration with field observations. Another limitation of using $\omega$ as the scale parameter is that the discreteness (integer values) of this parameter inherently limits the "detail" with which scaling properties of a river basin can be examined, and also the characterization of these properties beyond average quantities. For example, Peckham and Gupta [1999] attempted generalization of Horton's laws to study the scaling of whole probability density functions (PDFs) of basin attributes. They used empirical methods to compare cumulative distribution functions and demonstrated simple scaling, meaning that the PDFs can be rescaled from one stream order to another with a single scaling exponent. However, the discrete nature of stream order and the small number of subbasins of order greater than 3 precluded an accurate analysis of multiscaling properties. Yet, development and testing of realistic highresolution landscape evolution models requires more detailed and complete description of the spatial heterogeneity present in landscapes (beyond first-order statistical moments) and better understanding of the processes that drive the observed heterogeneity.

[6] The HS stream ordering system is typically applied only in the fluvial portion of landscapes and scaling relationships that might exist in unchannelized areas or zeroorder basins, are not typically considered, greatly inhibiting study of the continuum of the hillslope-fluvial system. Previous research has documented that unchannelized regions can be divided into a network of hollows, divergent hillslope nose, and side slopes [Hack and Goodlett, 1960]. Hillslope hollows have been observed to be organized into distinct, quasi-connected flow paths [Dietrich et al., 1987] that exhibit different topological characteristics compared to the fluvial network. In terms of Horton's laws, this regime has been documented as exhibiting higher $R_{B}$ and higher $R_{A}$ ratios along the hillslope compared to fluvial networks [Hack and Goodlett, 1960]. The HS ordering scheme is also limited in that it is difficult to apply quantitatively to many physical, chemical, and biological processes that are more directly related to characteristic length scales. Stream metabolism and nutrient spiraling [Finlay et al., 2002; Lowe et al., 2006], flood pulse propagation or attenuation [Moussa and Bocquillon, 1996; Junk, 1999], and gravel bed load transport [Schmidt and Ergenzinger, 1992] are just a few examples of processes that are loosely related to stream order, but are more properly defined in terms of length scale.

[7] Rinaldo and Rodriguez-Iturbe [1998] emphasized the limited power of Horton's laws in discriminating among different branching network topologies [see also Kirchner, 1993] and proposed using length or area-based ordering schemes. For example, Rigon et al. [1996] used contributing area as the scale parameter to compute higher-order statistical moments of stream lengths, measured between a given point in the channel network and its corresponding drainage divide, and demonstrated the existence of simple scaling. Using $30 \mathrm{~m}$ DEMs they confined their scaling analysis only within the fluvial part of the landscape.

[8] With increasing availability of high-resolution topography data from lidar and ever increasing computational processing capability, we are now in a position to begin studying the detailed spatial organization of landscapes more quantitatively, and seamlessly across hillslopes and channels, at very fine $(<1 \mathrm{~m})$ resolution. The present paper is a step in this direction and, building on previous developments, introduces two main innovations. First, we propose the use of "directed distance from the divide," $\ell$, as the scale parameter (instead of Horton's stream order or upstream contributing area) and demonstrate its ability to probe down to zero-order unchannelized basins and quantify the statistical heterogeneity of slopes, curvatures and upstream contributing areas moving seamlessly across the hillslope and fluvial regimes. Second, we show that topographic attributes conditioned on directed distance from the divide exhibit less variability than those same attributes conditioned on upstream contributing area, affording thus a sharper identification of regime transitions and increased accuracy in the scaling analysis.

[9] The paper is structured as follows. In section 2 we define directed distance from the divide and discuss its use as the scaling parameter. In section 3 we use simulated networks to demonstrate that when the law governing the flow path arrangement is the same at all scales, a single scaling regime is found with a scaling exponent which directly relates to Horton's parameters. In section 4 we analyze three nested river basins in northern California and demonstrate the ability of the proposed framework to depict transitions in geomorphic regime. In section 5, we quantify in detail the statistical scaling properties in each regime pointing to the distinctly different degrees of spatial heterogeneity in each regime. Finally, in section 6 we discuss the advantage of using directed distance from the divide, compared to contributing area, as the scale parameter for topographic attributes (e.g., slope) and show that the former results in less variability, allowing thus an easier depiction of regime transitions and a tighter statistical characterization. Concluding remarks are made in section 7.

\section{Directed Distance From the Divide as the Scale Parameter}

[10] Directed distance from the divide $(\ell)$ is defined as the length from a given pixel in the landscape to the drainage divide measured along the longest topographically delineated flow path. When two flow paths converge, the shortest upstream flow path is terminated and the flow distance continues along the main path. This definition is the same as 

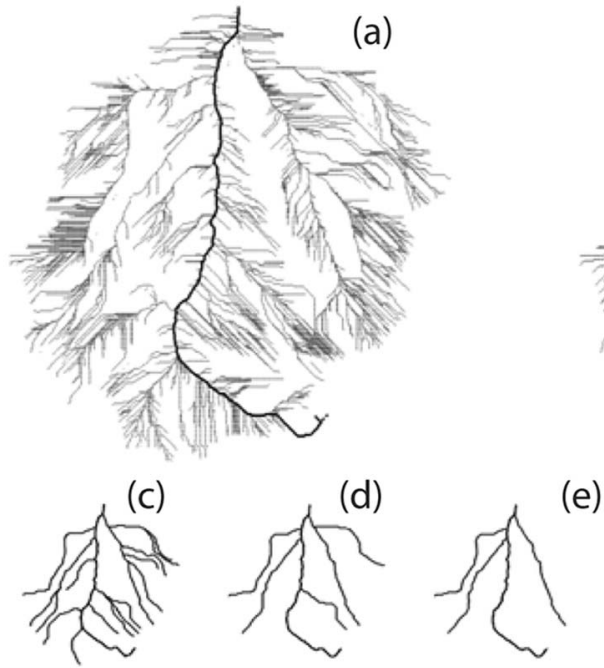
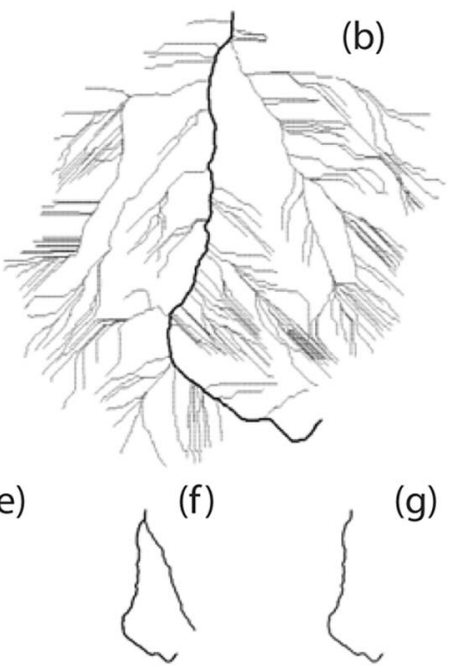

Figure 1. A new stream ordering system based on flow path distance $\ell$ called "directed distance from the divide." (a) Basic network of flow path segments for $\ell>50 \mathrm{~m}$. (b) Flow path segments with $\ell<100 \mathrm{~m}$ are eliminated from Figure 1a at their corresponding tributary junctions. (c) Flow path segments with $\ell<200 \mathrm{~m}$ are eliminated from the network at their tributary junctions. Flow path segments $\ell$ less than (d) $300 \mathrm{~m}$, (e) $400 \mathrm{~m}$, (f) $500 \mathrm{~m}$, and (g) $600 \mathrm{~m}$.

that used by Rigon et al. [1996], who, however, adopted the upstream contributing area as the scale parameter to study the statistical characteristics of directed distance from the divide; differences and similarities with that study will be discussed more extensively in sections 3,5 , and 6 .

[11] To compute directed distance, we assign a value to every pixel within a river basin according to their location along flow paths, beginning at pixels that receive no flow from neighboring pixels (i.e., flow accumulation is zero), which we consider source points. We use the D8 flow direction algorithm [O'Callaghan and Mark, 1984] to select the next pixel in the downstream direction. For a $1 \mathrm{~m}$ digital elevation model (DEM), we compute the distance from pixel $i$ to pixel $j$ as $1 \mathrm{~m}$ if the flow is along a row or column or $\sqrt{ } 2 \mathrm{~m}$ if flow proceeds along a diagonal path. If flow is directed into pixel $j$ from another pixel $k$, the flow path with the higher length value at pixel $j$ is used and the shorter flow path terminates at pixel $i$ or $k$. The algorithm for extracting directed distance keeps track of all pixels along all flow paths distributed throughout the basin and therefore permits analysis of ensemble statistics of geomorphic attributes at any value of $\ell$. For the purpose of illustration, Figure 1 demonstrates the method and defines the flow path topology for different values of $\ell$, from $\ell=50 \mathrm{~m}$ to $\ell=600 \mathrm{~m}$ for a tributary basin in the TR6 basin. We assume that the set of points on the ridge lines that initiate the directed distance $\ell$ flow paths define the significant divides at scale $\ell$.

[12] Natural landscapes are subject to variability in environmental conditions (e.g., geology, vegetation, and soil type) and therefore spatial heterogeneity in morphological attributes for a given value of $\ell$ is expected to exist. Using directed distance from the divide as the scale parameter, one can zoom down to an increasing range of scales $\ell$ and assess how the PDFs of morphological attributes change as $\ell$ changes within or across river basins. We consider the PDF of any basin attribute $\theta$ (e.g., contributing area, number of flow paths, local slope, or curvature), $P(\theta(\ell))$, for a given value of the scale parameter $\ell$ and denote by $\langle\theta(\ell)\rangle$ the ensemble average of the attribute (ensemble average area, slope, or curvature, or summation of the number of flow paths etc.) at distance $\ell$. We also compute higher-order statistical moments of those attributes, $\left\langle\theta(\ell)^{q}\right\rangle$, and use them to quantify how the entire PDF changes as a function of scale.

\section{Scaling Relationships in Simulated Networks}

[13] In this section, we consider a number of synthetic networks in order to illustrate that using directed distance from the divide as the scale parameter correctly depicts the expected scaling relationships and that no scaling break is found since the generating rule is the same at all scales. We consider two general classes of synthetic networks: branching tree graphs and spanning tree graphs. Branching tree graphs are line graphs that simply represent the topological arrangement of stream segments, so they differ from spanning trees in that they do not simulate an entire basin [Manna and Subramanian, 1996; Rinaldo and RodriguezIturbe, 1998; Banavar et al., 1999; Banavar et al., 2001; Flammini and Colaiori, 1996]. Simple network models such as these are useful to demonstrate the essential statistical properties that can also be observed in real river networks. Spanning tree graphs are computed from grids that cover an entire computational domain without forming loops, such as to simulate transport of water or sediment downstream from source areas to the basin outlet.

[14] Two key parameters can be defined to quantify the flow path arrangement. The first parameter, $\gamma$, is the exponent of the power law decay relationship between the scale parameter $\ell$ and the number of streams that exist at a given value of the scale parameter, $N(\ell)$, [see also Rigon et al., 1998]:

$$
N(\ell) \propto \ell^{-\gamma}
$$


This parameter applies to both branching and spanning trees. The expected value of $\gamma$ can be written as

$$
\gamma=\frac{\log R_{B}}{\log R_{L s}}
$$

This implies that using $\ell$ as the scale parameter one combines the Hortonian length and bifurcation ratios into a new scaling exponent $\gamma$. The second parameter, $H$, is derived from Hack's law [Hack, 1957]. This parameter describes the relationship between flow path length $\ell$ and its average upstream contributing area $\langle A(\ell)\rangle$ and is therefore only applicable to spanning trees, which account for area within the computational domain. This relation is not exactly the same as Hack's law, but can be reconciled with Hack's law as

$$
\langle A(\ell)\rangle \propto \ell^{H}
$$

where $1 / H$ is approximately equal to the Hack exponent. The expected value of $H$ is

$$
H=\frac{\log R_{A}}{\log R_{L s}}
$$

Once again, we observe that using $\ell$ as the scale parameter combines the Hortonian area and length ratios into a single exponent $H$.

\subsection{Branching Trees}

[15] Branching tree networks are among the simplest of network models defined by the topological arrangement of stream segments. The simplest type of branching tree network is a binary tree [e.g., see Shreve, 1966; Dodds and Rothman, 2000a], in which two stream segments join at a junction and generate a downstream segment. A perfect binary tree is an idealized Hortonian network that assumes symmetry in branching, and in which only equivalent stream orders join (i.e., two first-order streams come together to form a second order, and two second-order streams come together to form a third order and so on, but a first-order stream never flows into a third-order stream). Along the network, $\omega$ increases continuously up to the highest-order $\Omega$.

[16] Figure 2 (top left) shows a synthetic binary tree where the depth of the tree extends up to ten levels. We maintain the length ratio of the binary tree $R_{L}$ equal to 2 and the angle between two links equal to $30^{\circ}$. This results in a bifurcation ratio $R_{B}$ of 2 for our binary tree network, which is much lower than that typically observed in real river networks. Simple ternary trees (Figure 2, top right), where three segments of the same order come together at each junction have $R_{B}=3$, but such orientations are not common in natural fluvial networks. Rather, the higher bifurcation ratio observed in natural fluvial networks (typically between 3 and 5) is due to the side tributaries that join with a trunk channel of higher order, and therefore do not change the order of the trunk channel. For the binary and ternary branching trees the scaling exponent $\gamma$ does not change as a function of scale because the rules governing bifurcation are consistent throughout the domain. Taken as the exponent of the power law relationship of $N(\ell)$ as a function of $\ell$ (equation (2)), $\gamma$ is constant at 1.0 and 1.59 for binary and ternary trees, respectively (see Table 1). It is noted that when performing computations using $\ell$ (directed distance from the divide) or $\ell_{s}$ (the Hortonian segment length), both approaches converge to the same value of $\gamma$, apart from small differences at small scales, as shown in Figure 2.

\subsection{Spanning Trees}

[17] Spanning tree graphs add one layer of complexity on top of the branching trees in that the entire spatial domain is considered. We use a Scheidegger directed random network model [Scheidegger, 1967; Takayasu et al., 1988; Huber, 1991; Marani et al., 1991; Nagatani, 1993a, 1993b; Rinaldo and Rodriguez-Iturbe, 1998; Banavar et al., 1999] as one type of spanning tree graph to illustrate the computation of the scaling parameters $\gamma$ and $H$ in these models that apply a simple set of rules throughout the entire domain. The Scheidegger network is developed according to the simple rules that water flows from the top to the bottom of the domain and flow direction at every pixel is chosen randomly between two diagonal downstream directions with 0.5 probability. In this model, once flow paths converge, they are not permitted to diverge downstream. For the purpose of measuring $\ell$, upon convergence of two flow paths, the shorter flow path is terminated and water and sediment from that shorter flow path are contributed to the stream with the longer upstream flow path. Periodic boundary conditions are imposed on east and west boundaries, such that water flowing off one side of the grid reenters the grid on the opposite side in the appropriate row.

[18] Figures $3 \mathrm{a}$ and $3 \mathrm{~b}$ show the estimation of the scaling parameters $\gamma$ and $H$ using directed distance from the divide. It is observed that (1) the estimated parameters are constant for the entire range of directed distances because the rules governing flow path arrangement do not change and (2) the estimated values of the exponents are consistent with the expected theoretical values. To quantify the way in which the PDF of contributing area changes as a function of the scale parameter $(\ell)$ we consider higher-order statistical moments:

$$
\left\langle A(\ell)^{q}\right\rangle \propto \ell^{\tau(q)}
$$

where $q$ is the order of the moment and $\tau(q)$ is the spectrum of scaling exponents which can be estimated from the slope of the statistical moments plotted against the scale $\ell$ in a $\log -\log$ plot. When $\tau(q)$ is a linear function of $q$, the relationship can be written as $\tau(q)=q(H)$, where $H$ is the scaling exponent that can be used to rescale the PDF from one scale $\ell$ to another scale $\lambda \ell$, where $\lambda$ is a constant. A linear relationship between $\tau(q)$ and $q$ is referred to as simple scaling. However, $\tau(q)$ is often found to be a nonlinear function of $q$ (referred to as multiscaling), implying a more complex renormalization of statistical moments which needs more than one parameter to be quantified.

[19] Figure 3c shows the higher-order statistical moments of contributing area $\left\langle A(\ell)^{q}\right\rangle$ for $q=0.5$ up to 5 at 0.5 intervals as a function of directed distance from the divide for the Scheidegger network. Each statistical moment plots linear in log-log space, the slope of which is used to develop the $\tau(q)$ curve shown in Figure 3d. The linear relationship observed between $\tau(q)$ and $q$ indicates simple scaling in the way the PDF of area changes as a function of directed distance. Simple scaling is to be expected in a basin where 

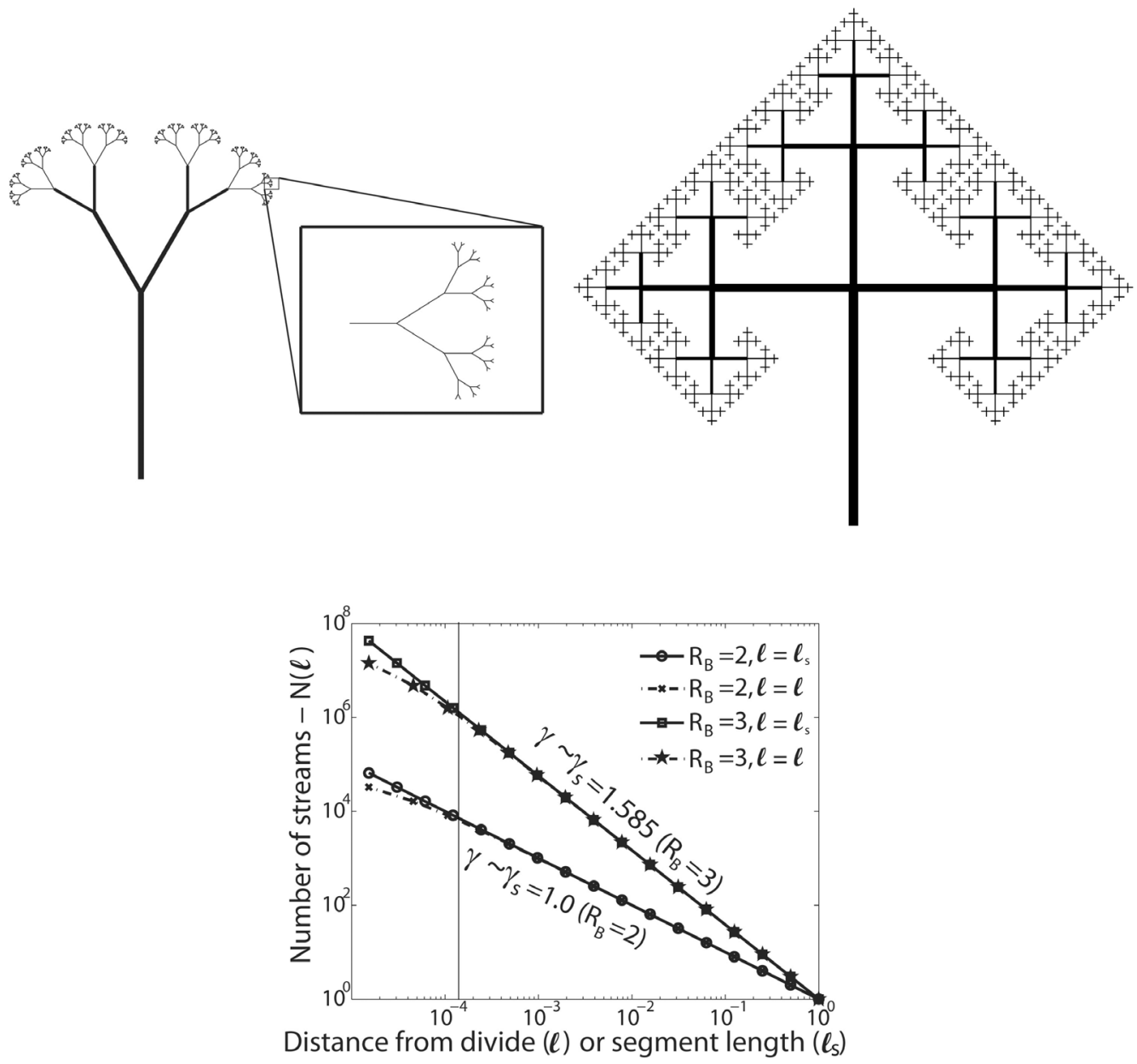

Figure 2. (top left) $\mathrm{A}$ binary tree with $R_{B}=2$ and $R_{L s}=2$ and branching angle of $30^{\circ}$. (top right) A ternary tree with $R_{B}=3$ and $R_{L s}=2$ and branching angle of $90^{\circ}$. (bottom) The number of streams with length greater or equal to a given distance from the divide is plotted against the normalized segment length $\left(\ell_{s}\right)$ and the total normalized distance from the divide $(\ell)$. It is seen that a single scaling regime is observed and that the estimated values of $\gamma$ agree with the expected theoretical values computed from equation (3) except at very small scales (approximately four levels as indicated by the vertical line) where the segment length $\left(\ell_{s}\right)$ and total length $(\ell)$ differ slightly.

the rules governing flow path arrangement do not change throughout the domain.

\section{Depicting Regime Transitions in Real Basins}

[20] In the synthetic networks considered in section 3, we observed no scaling breaks because the rules governing flow accumulation and flow path topology are consistent throughout the domain. However, as documented below, in real landscapes we observe distinct scaling breaks that correspond to transitions in flow path topology and, by inference, in the geomorphic process giving rise to that topology. In the hillslope domain, between the drainage divides and the stream network, the distributions of several topographic attributes (e.g., slope, curvature, and upstream contributing area) change at different rates as a function of scale compared to those in the fluvial part of the network. The question as to which topographic attributes might exhibit the greatest sensitivity to differences in geomorphic regime, and therefore can most effectively be used to identify regime transitions, is of basic and practical interest. To address this question we analyze changes in the number of flow paths and the distributions of slopes and curvatures as a function of our scale parameter $\ell$. We also compare those results to the same analysis using the upstream contributing area as the scale parameter similarly to the analysis of Rigon et al. [1996].

Table 1. Comparison of Parameters in Simulated River Networks ${ }^{\mathrm{a}}$

\begin{tabular}{lccccccccc}
\hline \multirow{2}{*}{ Network } & $\begin{array}{c}\text { Scaling } \\
\text { Range (m) }\end{array}$ & $R_{B}$ & $R_{L s}$ & $R_{A}$ & $\frac{\log R_{B}}{\log R_{L s}}$ & $\frac{\log R_{A}}{\log R_{L s}}$ & $\gamma$ & \\
\hline Binary & $1-\ell_{\max }$ & 2.0 & 2.0 & - & 1.000 & - & 1.0 & - \\
Ternary & $1-\ell_{\max }$ & 3.0 & 2.0 & - & 1.585 & - & 1.6 & - \\
Scheidegger & $1-\ell_{\max }$ & - & - & - & - & - & 1.3 & 1.5 \\
\hline
\end{tabular}

${ }^{\mathrm{a}} R_{B}$ and $R_{L s}$ are Horton's ratios (see equations (1a) and (1b)); (log $R_{B} /$ $\left.\log R_{L s}\right)$ and $\left(\log R_{A} / \log R_{L s}\right)$ are the theoretically expected values of the scaling exponents $\gamma$ and $H$; and $\gamma$ and $H$ are the empirically estimated values. 

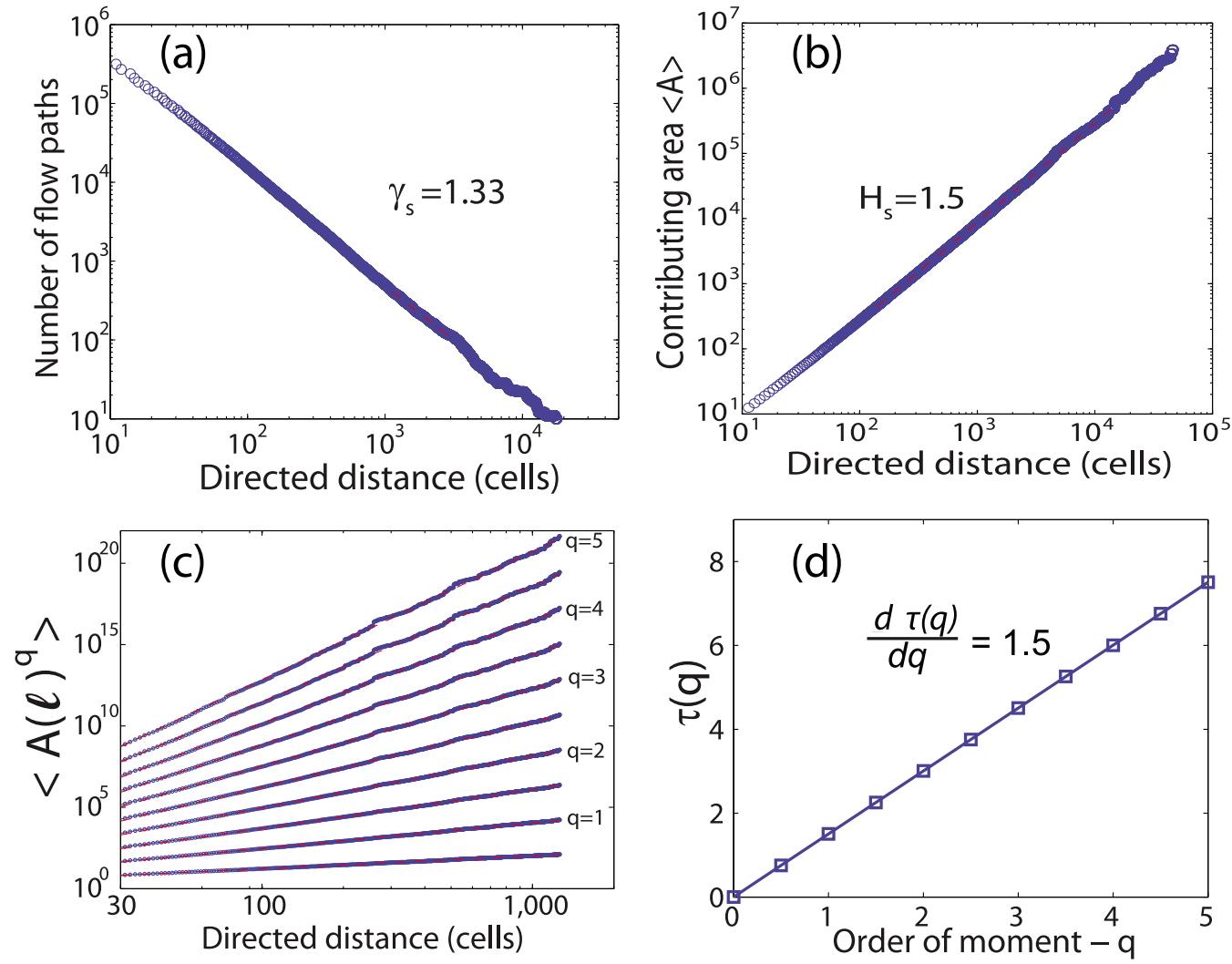

Figure 3. Scaling relationships for the Scheidegger network on a 33,000 $\times 2400$ grid lattice. (a) Number of flow paths versus directed distance from the divide. The exponent $\gamma$ of the power law characterizes flow path convergence. (b) Average upstream contributing area plotted against directed distance from the divide. The scaling exponent $H$ quantifies the accumulation of contributing area as a power law function of flow path distance. (c) Moments of order $q=0.5-5$, by 0.5 increments, of contributing area versus distance from the divide. (d) The spectrum of scaling exponents $\tau(q)$, computed from the slopes of the $\log$ - $\log$ plots of Figure 3c. It is observed that $\tau(q)=q H$, implying simple scaling, where $H$ equals 1.5 .

[21] Using the D8 flow accumulation algorithm on highresolution topography data we delineated the flow path network throughout the entire basin, including hillslopes. Although we recognize that the flow paths generated by the D8 algorithm might not be exactly the actual overland flow paths on hillslopes, the scaling analysis presented herein is shown to be robust in that it clearly establishes physically relevant regime transitions and correctly depicts the increased heterogeneity in the geomorphic attributes in the unchannelized part of the river basin, compared to the channelized part.

[22] We selected three nested basins located in Mendocino County, California, including the South Fork Eel River basin (SF Eel), Elder Creek basin, which is a tributary to SF Eel, and TR6, which is a tributary to Elder Creek (Figure 4). The Horton ratios for the three river networks (delineated using an area threshold of $10^{5} \mathrm{~m}^{2}$ ) are given in Figure 5 (see also Table 2). The nested basins allow us to examine the statistics of the same landscape at three sequential scales of observation. The basins have drainage areas of 354,18 , and $3 \mathrm{~km}^{2}$ and are underlain by Tertiary-Cretaceous Coastal Belt Franciscan clastic sedimentary rocks, primarily composed of arkosic sandstone, pebble conglomerate, and mudstone. We used the lidar topographic data $(1 \mathrm{~m}$ grid resolution, $0.3 \mathrm{~m}$ vertical accuracy) for the SF Eel collected in June 2004 and processed by the National Center of Airborne Laser Map-

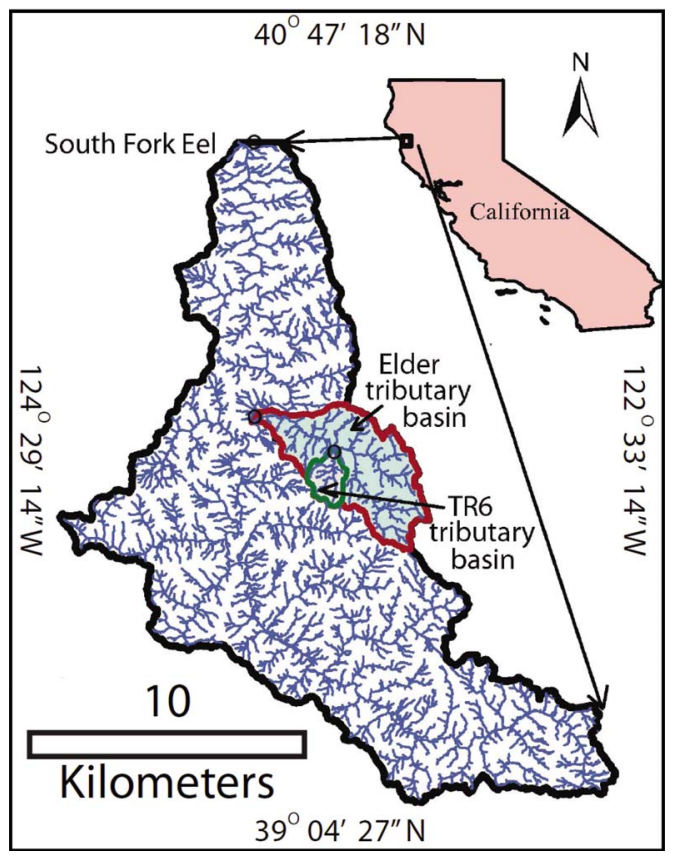

Figure 4. Three nested river basins in northern California used in our analysis: South Fork Eel River $\left(354 \mathrm{~km}^{2}\right)$, Elder Creek $\left(18 \mathrm{~km}^{2}\right)$, and TR6 $\left(3 \mathrm{~km}^{2}\right)$. 


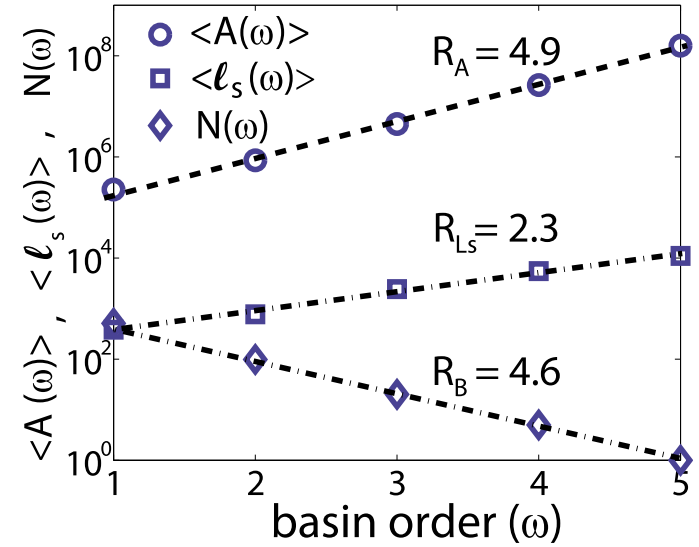

Figure 5. Horton's ratios for the South Fork Eel River. The logarithmic slope of the number of streams as a function of stream order $\omega$ is the bifurcation ratio, $R_{B}=4.6$ (diamonds). The logarithmic slope of the ensemble average of the contributing area as a function of stream order $\omega$ is the area ratio, $R_{A}=4.9$ (circles). The length ratio is computed as the logarithmic slope of the ensemble average of stream lengths as a function of stream order $\omega$, which yields $R_{L s}=$ 2.3 (squares).

ping (NCALM). The data are freely available for download at http://www.ncalm.org.

\subsection{Number of Flow Paths}

[23] Within each of the three study basins we computed the number of flow paths $N(\ell)$ that have distance from their respective drainage divides equal to or greater than $\ell$. Figure 6 shows plots of $N(\ell)$ versus $\ell$ for the three basins. Three distinct zones in flow path topology, which we refer to as regions $\mathrm{A}, \mathrm{B}$, and $\mathrm{C}$ are observed in each plot. The changes in slope observed in these plots, contrary to what we observed in the synthetic networks, are indicative of a transition in geomorphic process, that is to say, a change in the rules governing flow and sediment transport. Regions A and $\mathrm{B}$ physically reside in the unchannelized part of the basins and region $\mathrm{C}$ marks the transition to the fluvial network, based on visual inspection. Region A does not exhibit power law scaling throughout the region. The nonlinear logarithmic change in the number of flow paths as a function

Table 2. Comparison of Scaling Parameters in Three California River Basins

\begin{tabular}{|c|c|c|c|c|c|c|c|c|}
\hline Network & $\begin{array}{c}\text { Scaling } \\
\text { Range (m) }\end{array}$ & $R_{B}$ & $R_{L s}$ & $R_{A}$ & $\frac{\log R_{B}}{\log R_{L s}}$ & $\frac{\log R_{A}}{\log R_{L s}}$ & $\gamma$ & $H$ \\
\hline \multicolumn{9}{|c|}{ South Fork Eel River } \\
\hline Region A & $1-79$ & - & - & - & - & - & & 1.00 \\
\hline Region B & $80-250$ & - & - & - & - & - & 2.87 & 3.06 \\
\hline Region $\mathrm{C}$ & $270-6000$ & 4.6 & 2.3 & 4.9 & 1.83 & 1.91 & 2.21 & 1.80 \\
\hline \multicolumn{9}{|l|}{ Elder Creek } \\
\hline Region A & $1-83$ & - & - & - & - & - & - & 1.01 \\
\hline Region B & $83-322$ & - & - & - & - & - & 3.15 & 3.01 \\
\hline Region C & $323-1500$ & 4.4 & 2.2 & 5.1 & 1.87 & 2.07 & 2.22 & 1.85 \\
\hline \multicolumn{9}{|c|}{ TR6 Tributary Basin } \\
\hline Region A & $1-80$ & - & - & - & - & - & - & 1.05 \\
\hline Region B & $81-270$ & - & - & - & - & - & 3.12 & 3.01 \\
\hline Region $\mathrm{C}$ & $271-1500$ & 4.1 & 2.1 & 4.9 & 1.901 & 2.14 & 2.12 & 1.87 \\
\hline
\end{tabular}

of $\ell$ in region $A$ is indicative of the divergent or parallel flow path topology in the upper, creep-dominated portions of hillslopes. The scaling exponent $\gamma$ varies from 2.87 to 3.15 and 2.12 to 2.22 for regions $\mathrm{B}$ and $\mathrm{C}$, respectively. In the SF Eel basin, scaling regions $\mathrm{B}$ and $\mathrm{C}$ extend from 80 to $250 \mathrm{~m}$ and 270 to $6000 \mathrm{~m}$, respectively (Table 2). It is remarkable that the trends are so similar in the three basins which span a wide range of sizes.

[24] Table 2 summarizes the computed values of $R_{B}, R_{L S}$, and $R_{A}$ (Horton's ratios) for the three networks as well as the expected values (from equation (2)) and estimated values of $\gamma$ from the $\log -\log$ plots of Figure 6 . It is noted that the rate of removal of flow paths is greater in region $\mathrm{B}$ than in region
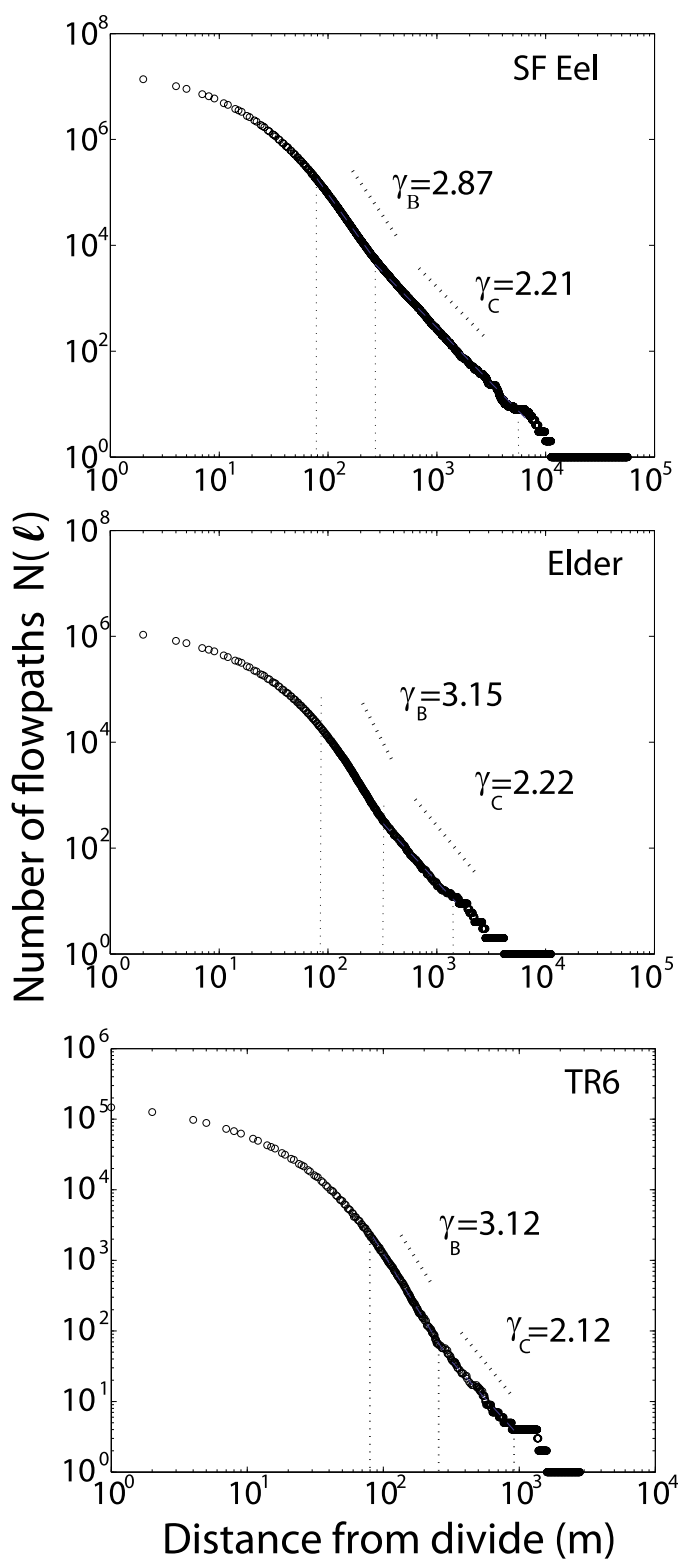

Figure 6. Number of flow paths as a function of directed distance from the divide in three tributary basins, (top) South Fork Eel River, (middle) Elder Creek, and (bottom) TR6. Three statistically distinct regions are observed in all three basins. The exponents of the power law relationships for regions $\mathrm{B}$ and $\mathrm{C}$ are shown in the plots. 


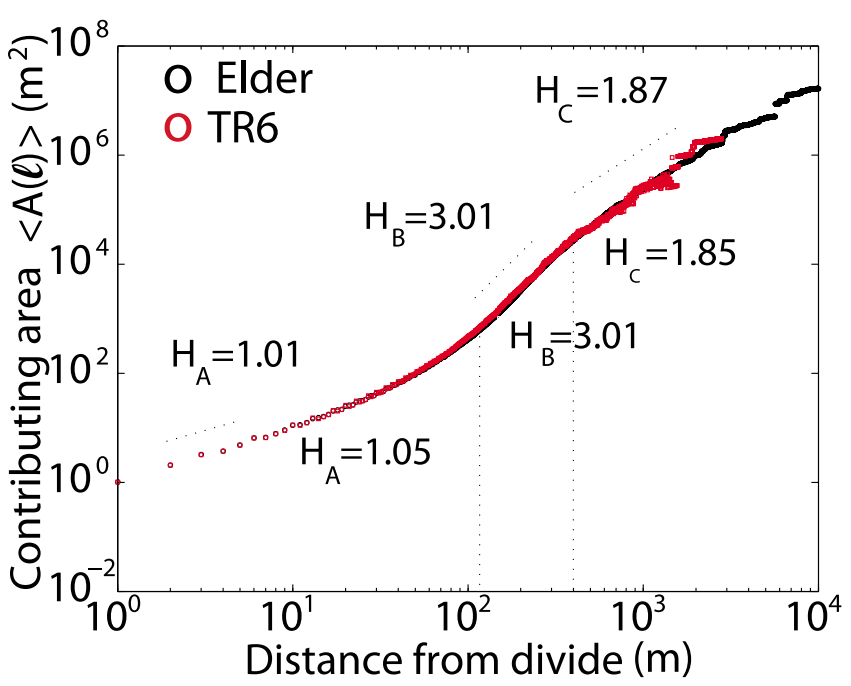

Figure 7. Ensemble-average contributing area for Elder Creek and TR6 shown against the scale parameter $\ell$ measured from drainage divides. Three scaling regions are observed as indicated by the vertical dashed lines from 1 to $50 \mathrm{~m}, 100$ to $300 \mathrm{~m}$, and 400 to $\ell_{\max } \mathrm{m}$, where $\ell_{\max }$ is the mainstream length.

$\mathrm{C}$, as indicated by the relatively higher exponent in hillslope region $\mathrm{B}, \gamma_{B}$, compared to that of the fluvial region, $\gamma_{c}$, in all three basins (Table 2), indicating that region $\mathrm{B}$ is the most convergent part of the landscape.

\subsection{Contributing Area, Slope, and Curvature}

[25] For all points in the basin at distance $\ell$ from the divide, we computed the upstream contributing areas, the local slopes and local (Laplacian) curvatures. Figure 7 shows the ensemble mean contributing area as a function of directed distance from the divide. Three power law scaling regimes can be observed which coincide with the scaling regimes seen in the number of flow paths plot of Figure 6 (see also Table 2). The scaling exponent $H$ for region $\mathrm{A}$ is nearly one, indicating that in this region, the contributing area grows at essentially the same rate as distance from the divide, implying almost parallel flow paths. The highest scaling exponent occurs in region $\mathrm{B}$, where area increases as length to the third power. Region $\mathrm{C}$ exhibits a scaling exponent of 1.8, consistent with Hack's law for fluvial networks.

[26] Using the directed distance as the scale parameter we also studied the organization of landscape attributes such as local slope $S$ and curvature $C$. Figure 8 shows the ensemble averages of slope $\langle S\rangle$, curvature $\langle C\rangle$, and the slope-curvature relationship when both parameters are conditioned on $\ell$. Again, all three landscape attributes exhibit three distinct scaling regions that coincide reasonably well with the scaling breaks observed in Figures 6 and 7, further confirming the distinctly different flow path topologies in the channelized and unchannelized parts of a basin. An important finding here is that the transitions are most abrupt in Figure $8 \mathrm{c}$ (slope versus curvature), indicating that there are systematic differences in the way slope and curvature respond to changes in geomorphic regime. Exploiting these
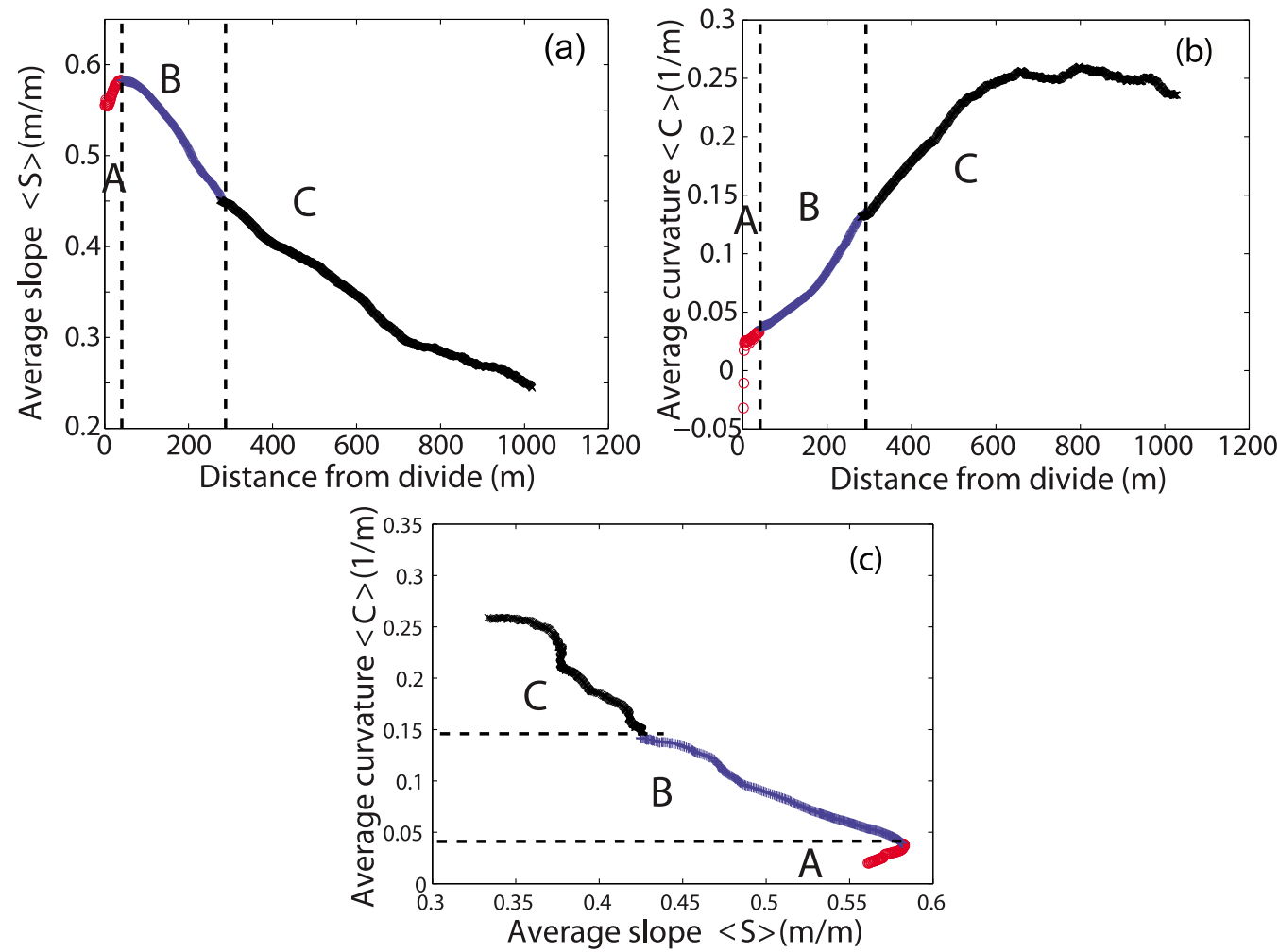

Figure 8. The ensemble-average (a) slope, (b) curvature, and (c) slope-curvature relationships for Elder Creek. The distinct regions can be demarcated as A, B, and C on the basis of the changing trend of the attributes as a function of $\ell$. These regions are consistent with those extracted from the number of flow paths and contributing area plots of Figures 6 and 7. 

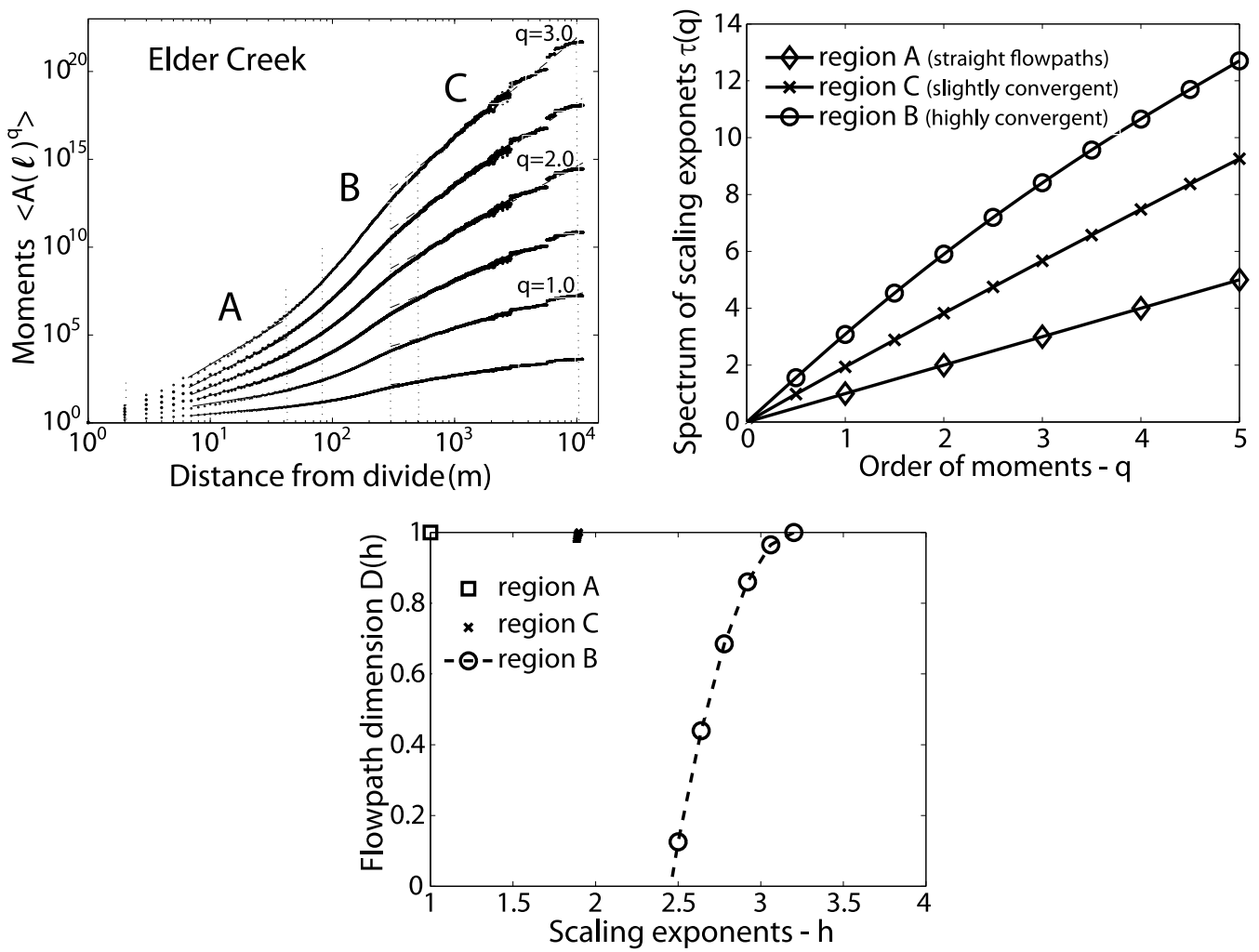

Figure 9. (top left) Higher-order structure functions, (top right) scaling exponent spectrum, and (bottom) $D(h)$ spectrum for regions $\mathrm{A}, \mathrm{B}$, and $\mathrm{C}$ for contributing area $\mathrm{A}(\ell)$ for Elder Creek. The structure functions exhibit three distinct scaling regimes as marked by dotted lines: region A $(1-50 \mathrm{~m})$, region $\mathrm{B}$ $(100-300 \mathrm{~m})$, and region $C\left(400-\ell_{\max } \mathrm{m}\right)$. Scaling exponent spectrum is linear for region A and nearly linear for region $\mathrm{C}$. Region B shows nonlinear behavior of $\tau(q)$ as $q$ increases. The range of scaling exponents represented within each region is shown in the $D(h)$ spectrum (bottom plot). Note the wide multifractal spectrum observed in region B (an indication of the large spatial heterogeneity of flow path organization in that region) and the much narrower range (i.e., $h=[1.7,1.87]$ ) observed in the fluvial region $\mathrm{C}$.

differences appears to be the most effective means by which distinct regions can be delineated. This observation should be explored further to determine if the same trend is replicated in other landscapes.

\section{Quantifying Spatial Heterogeneity in Landscape Organization}

[27] In Figure 7 the scaling of average contributing area as a function of directed distance from the divide was documented and the scaling exponents in regions $\mathrm{A}, \mathrm{B}$, and $\mathrm{C}$ were reported. Going to higher-order statistical moments, Figure 9 (top left) reports moments of order $q=0$ to $q=3$ in increments of 0.5 for Elder Creek basin. Log-log linear relationships are observed between the scales marked with dotted lines. The scaling ranges for regions $\mathrm{B}$ and $\mathrm{C}$ are approximately $100-300$ and $500-10,000 \mathrm{~m}$, respectively. Fitting straight lines to all moments and computing the slopes results in the spectrum of scaling exponents $\tau(q)$ shown in Figure 9 (top right) for each regime. We recall that a linear $\tau(q)$ curve results in a single scaling exponent $H$ $(H=d \tau(q) / \mathrm{d} q)$ which can be used to renormalize the entire PDF (all statistical moments) of contributing area as a function of directed distance from the divide. However, a nonlinear $\tau(q)$ curve results in a range of scaling exponents summarized in the $D(h)$ spectrum, where $D(h)$ is computed from $\tau(q)$ using the Legendre transform [Parisi and Frisch, 1985; Muzy et al., 1994]:

$$
D(h)=\min _{q}[q h-\tau(q)+1]
$$

The $D(h)$ spectrum summarizes the range of fractal exponents $(h)$ needed to rescale the PDF of contributing area for different values of $\ell$.

[28] The $D(h)$ spectrum for regions $\mathrm{A}, \mathrm{B}$, and $\mathrm{C}$ is shown in Figure 9 (bottom). In region A, a single scaling exponent $H=1$ is observed consistent with the almost parallel flow paths in those parts of the landscape that are close (within $100 \mathrm{~m}$ length scale) to the divides. However, in region B, extending from 100 to $400 \mathrm{~m}$, hillslopes exhibit a wide $D(h)$ spectrum indicating that multiple exponents are needed to rescale the PDF of contributing area as a function of $\ell$ in the downstream direction along the flow paths. This suggests that a larger spatial heterogeneity of flow path arrangement exists in region $\mathrm{B}$, requiring a more complex rescaling transformation in that part of the landscape. The dominant scaling exponent in this region is approximately 3.2. In the fluvial region $\mathrm{C}$ we observe a very narrow $D(h)$ spectrum where $h$ ranges between 1.7 and 1.87. (Note that these 


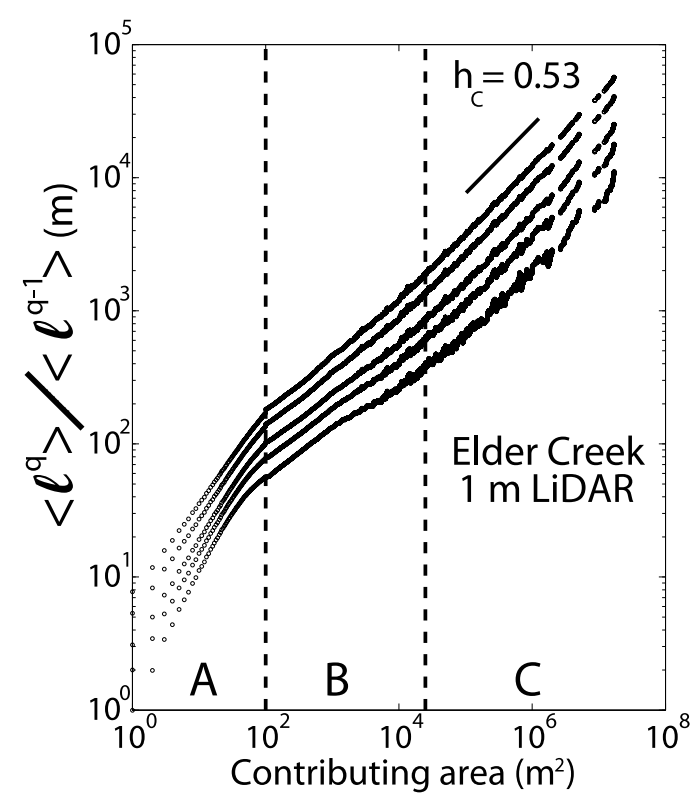

Figure 10. Application of the statistical framework of Rigon et al. [1996] using $1 \mathrm{~m}$ data in Elder Creek. Note the abrupt transition consistent with the scaling break observed between regions $\mathrm{A}$ and $\mathrm{B}$ using directed distance from the divide. The scaling break between regions B and C is observed, but the transition is more diffuse compared with Figure 8c.

exponents correspond to values of $0.53-0.59$ for the Hack's law exponents consistent with what is expected in the fluvial regime). We note that the above results agree with and extend beyond the fluvial regime the findings of Rigon et al. [1996], who documented monofractal behavior of flow path distances parameterized by contributing area in the fluvial part (region C) of river basins (generalization of Hack's law). Repeating here for completeness the analysis of Rigon et al. [1996] using $1 \mathrm{~m}$ data for the Elder Creek basin, we observe log-log linear scaling for region $\mathrm{C}$ with an exponent equal to 0.53 , consistent with Hack's law and with our results (see Figure 10). Two other scaling regions are also observed in Figure 10 corresponding to the regimes depicted by the directed distance analysis. One then wonders what, if anything, is gained in this case by using distance versus contributing area as the scale parameter. We argue that although the regime transitions are identified in both cases, directed distance offers a few distinct advantages for scaling topographic attributes, as discussed in section 6.

\section{Distance Versus Area as a Scale Parameter}

[29] A single parameter cannot be expected to be universally applicable for scaling the wide range of processes shaping landscapes and occurring in watersheds. But it is instructive to consider which processes are better scaled with area versus directed distance. In general, it should be expected that processes that depend exclusively on flow accumulation should be better scaled using upstream contributing area. Processes that are dependent on linear distances, such as hillslope creep, should be better scaled using directed distance.

[30] Two metrics can be used to argue that directed distance is a more relevant scaling parameter for topographic attributes within the hillslope domain, at least for the basins analyzed here. The first metric is the level of distinction with which the transitions between geomorphic regimes can be identified. As discussed in section 4.2 and observed in Figure 8 , the distinction between the geomorphic regimes is greatest in the plot of local slope versus local curvature when both variables are conditioned on directed distance from the divide.

[31] The second metric in quantifying the effectiveness of a scale parameter is the degree of variability observed in related basin attributes (e.g., slope) throughout a landscape conditioned on a specific value of the scale parameter. Lower variability in the related basin attributes would indicate greater suitability of the scale parameter. Figure 11 shows the coefficient of variation (standard deviation divided by the mean) of local slope as a function of distance from the divide, CV $S(\ell)$, and as a function of contributing area, CV $S(A)$, for Elder Creek basin and for distances $<1000 \mathrm{~m}$. Several important observations can be made from this plot. First, the CV $S(\ell)$ values are consistently lower than those of CV $S(A)$ for values of $\ell<700 \mathrm{~m}$, indicating that the spatial variability of slopes conditioned on $\ell$ is smaller compared to that conditioned on A throughout the entire hillslope domain and the upper fluvial domain. In simpler words, a hundred points in the landscape with the same upstream area can have significantly different local slopes (a large spread in the probability distribution of slopes for the same exact area), while a hundred points in the landscape with the same upstream length have a much narrower range of variability in their corresponding local slopes, allowing thus a more effective statistical characterization and a more physically relevant interpretation. Second, the local minimum near $\ell=70 \mathrm{~m}$ depicts the transition from near-parallel flow paths (region A) to highly convergent flow paths (region B). The CV $S(\ell)$ values exhibit much

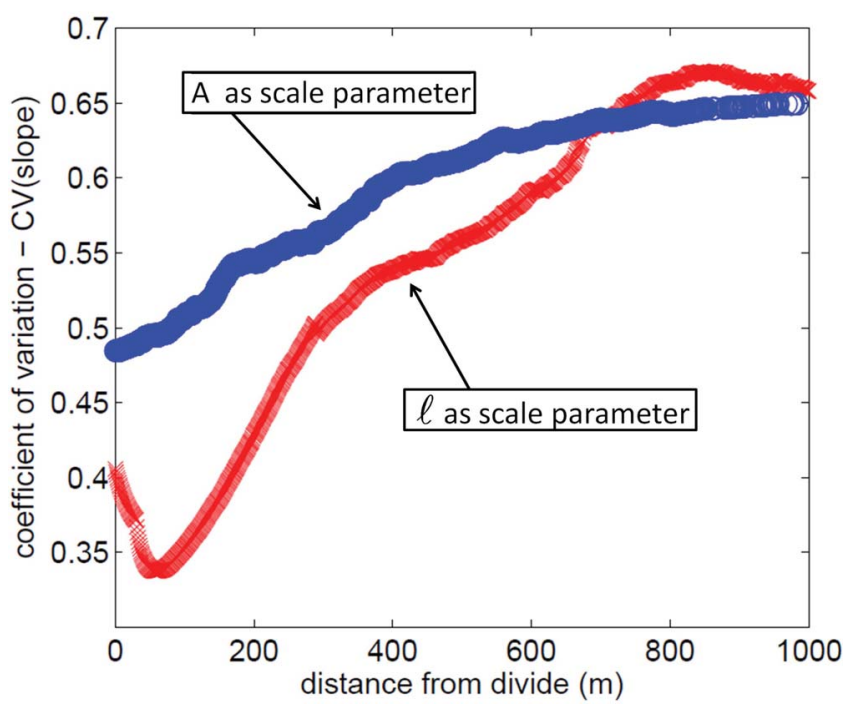

Figure 11. Coefficient of variation (CV) for local slope computed using directed distance as the scale parameter for $\ell \leq 1000 \mathrm{~m}$ (red) and using contributing area as the scale parameter (blue). It is observed that the CV values for slope computed using directed distance as a scale parameter are consistently lower than those computed using area as the scale parameter. 
higher sensitivity to that regime transition compared to the values of $\mathrm{CV} S(\mathrm{~A})$. Third, note that both directed distance and contributing area provide evidence of the large spatial heterogeneity in region $\mathrm{B}$ as witnessed by the increasing $\mathrm{CV}$ with increasing scale [see also Gangodagamage et al., 2007]. However, both $\mathrm{CV} S(\ell)$ and $\mathrm{CV} S(A)$ stabilize for $\ell>700 \mathrm{~m}$ to a constant value, which supports the finding of a reduced spatial heterogeneity (simple scaling) of geomorphic attributes in the fluvial regimes as also documented by Rigon et al. [1996].

\section{Concluding Remarks}

[32] Despite considerable progress over the past decades, a challenging topic in geomorphology still remains the development of methods to efficiently and comprehensively analyze high-resolution topography data in order to quantify spatial variability and organization laws in landscape attributes and make inferences about the hydrologic, geomorphologic and ecologic processes that imprinted their signature on that landscape.

[33] We have demonstrated that directed distance from the divide can be used as a scale parameter to understand how flow path topology and basin attributes are organized in both the hillslope and the fluvial portions of drainage basins. We used directed distance to extract information about flow path topology and the rate at which contributing area accumulates in the downstream direction in several synthetic networks. We demonstrated simple scaling relationships for these hypothetical landscapes where the rules governing flow do not change along the flow path continuum. In contrast, in real river basins we observed two scaling breaks (three scaling regions) in the relationships of the number of streams, accumulation of contributing area, local slope, and local curvature as a function of directed distance from the divide. The observed scaling breaks infer transitions in geomorphic processes that are likely driven by hydrology and sediment dynamics and, at a finer scale, likely influence and are influenced by, ecological dynamics. The scaling breaks are most clearly distinguished in a plot of ensemble averages of slope versus curvature when both attributes are conditioned on directed distance from the divide. These regime transitions are also observable using upstream contributing area as the scaling parameter, but using directed distance was shown to result in sharper transitions and less variability in the ensemble statistics of topographic attributes. This finding infers that the processes shaping hillslopes in our study landscape (regions A and B) are better defined by mechanics related to length scale (e.g., creep) rather than upstream accumulation of water (for which area is a proxy). Last, we apply the directed distance method within a multiscaling framework to quantify the way in which the whole probability distributions (and not only the average value) of contributing drainage area changes as a function of the scale parameter. We observe the highest degree of spatial heterogeneity of flow path organization within the hillslope zone (region B), as evidenced by the range of exponents needed to rescale the PDF of area for different values of the scale parameter $\ell$.

[34] The ability to understand and quantify the spatial heterogeneity of landscapes across the hillslope-fluvial continuum, as presented in this study, provides new opportunities to identify complex topographical and topological signatures left behind by the physical process that shaped the landscape. It also allows us to differentiate among physically distinct regimes and to extend scaling laws beyond average quantities and beyond river networks. Such detailed understanding of landscape organization might be helpful in guiding the development of more realistic landscape evolution models over a wider range of scales and over diverse climatic, ecologic and geological settings.

[35] Acknowledgments. We thank Collin Bode (NCED, UC Berkeley) for providing us with the lidar data. Discussions throughout the course of this work with William Dietrich are greatly appreciated. We also would like to thank Theodore Fuller for a thorough review of an earlier version of this manuscript and many useful suggestions. Finally, we thank Peter Sheridan Dodds for his useful suggestions and the Associate Editor, Andrea Rinaldo, for insightful comments that considerably improved the presentation and focus of this study. This work has been partially supported by the National Center for Earth-Surface Dynamics (NCED), a Science and Technology Center funded by NSF's Office of Integrative Activities under agreement EAR-0120914 and NSF CDI grant EAR-0835789, a doctoral dissertation fellowship to the first author by the graduate school of the University of Minnesota, and the Ling Professorship in Environmental Engineering to the senior author. Computer resources were provided by the Minnesota Supercomputing Institute, Digital Technology Center, at the University of Minnesota.

\section{References}

Abrahams, A. D. (1984), Channel networks: A geomorphological perspective, Water Resour. Res., 20(2), 161-188, doi:10.1029/WR020i002p00161.

Banavar, J. R., F. Colaiori, A. Flammini, A. Giacometti, A. Maritan, and A. Rinaldo (1997), Sculpting of a fractal river basin, Phys. Rev. Lett., 78(23), 4522-4525, doi:10.1103/PhysRevLett.78.4522.

Banavar, J. R., A. Maritan, and A. Rinaldo (1999), Size and form in efficient transportation networks, Nature, 399, 130-132, doi:10.1038/ 20144.

Banavar, J. R., Francesca Colaiori, A. Flammini, A. Maritan, and A. Rinaldo (2001), Scaling, optimality, and landscape evolution, J. Stat. Phys., 104, 1-48, doi:10.1023/A:1010397325029.

Dietrich, W., and T. Dunne (1993), Channel heads, in Channel Network Hydrology, edited by K. Beven and M. J. Kirkby, pp. 175-219, John Wiley, Chichester, U. K.

Dietrich, W. E., S. L. Reneau, and C. J. Wilson (1987), Overview: "Zeroorder basins" and problems of drainage density, sediment transport and hillslope morphology, in sediment transport and hillslope morphology, Proc. Int. Symp. Erosion Sediment. Pac. Rim, 165, 27-37.

Dodds, P. S., and D. H. Rothman (2000a), Scaling, universality, and geomorphology, Annu. Rev. Earth Planet. Sci., 28(1), 571-610, doi:10.1146/ annurev.earth.28.1.571.

Dodds, P. S., and D. H. Rothman (2000b), Geometry of river networks III. Characterization of component connectivity, Phys. Rev. E, 63, 016117, doi:10.1103/PhysRevE.63.016117.

Finlay, J. C., S. Khandwala, and M. E. Power (2002), Spatial scales of carbon flow in a river food web, Ecology, 83(7), 1845-1859, doi:10.1890/ 0012-9658(2002)083[1845:SSOCFI]2.0.CO;2.

Flammini, A., and F. Colaiori (1996), Exact analysis of the Peano basin, J. Phys. A Math. Gen., 29, 6701-6708, doi:10.1088/0305-4470/29/21/ 006.

Gangodagamage, C., E. Barnes, and E. Foufoula-Georgiou (2007), Scaling in river corridor widths depicts organization in valley morphology, Geomorphology, 91, 198-215, doi:10.1016/j.geomorph.2007.04.014.

Hack, J. (1957), Studies of longitudinal profiles in Virginia and Maryland, U.S. Geol. Surv. Prof. Pap., 294-B.

Hack, J. T., and J. C. Goodlett (1960), Geomorphology and forest ecology of a mountain region in the central Appalachians, U.S. Geol. Surv. Prof. Pap., 347, 66 pp.

Horton, R. (1945), Erosional development of streams and their drainage basins: Hydrophysical approach to quantitative geomorphology, Geol. Soc. Am. Bull., 56, 275-370, doi:10.1130/0016-7606(1945)56[275: EDOSAT]2.0.CO;2.

Huber, G. (1991), Scheidegger's rivers, Takayasu's aggregates and continued fractions, Physica A, 170(3), 463-470, doi:10.1016/0378-4371(91) 90001-S. 
Ijjasz-Vasquez, E., and R. L. Bras (1995), Scaling regimes of local slope versus contributing area in digital elevation models, Geomorphology, 12, 299-311, doi:10.1016/0169-555X(95)00012-T.

Junk, W. (1999), The flood pulse concept of large rivers: Learning from the tropics, Arch. Hydrobiol., 115(3), 261-280.

Kirchner, J. W. (1993), Statistical inevitability of Horton's laws and the apparent randomness of stream channel networks, Geology, 21, 591-594, doi:10.1130/0091-7613(1993)021<0591:SIOHSL>2.3.CO;2.

Lashermes, B., E. Foufoula-Georgiou, and W. Dietrich (2007), Channel network extraction from high resolution topography using wavelets, Geophys. Res. Lett., 34, L23S04, doi:10.1029/2007GL031140.

Lowe, W. H., G. E. Likens, and M. Power (2006), Linking scales in stream ecology, BioScience, 56(7), 591-597, doi:10.1641/0006-3568(2006) 56[591:LSISE]2.0.CO;2.

Manna, S. S., and B. Subramanian (1996), Quasirandom spanning tree model for the early river network, Phys. Rev. Lett., 76(18), 3460-3463, doi:10.1103/PhysRevLett.76.3460.

Mantilla, R., V. K. Gupta, and O. J. Mesa (2006), Role of coupled flow dynamics and real network structures on Hortonian scaling of peak flows, J. Hydrol., 322(1-4), 155-167, doi:10.1016/j.jhydrol.2005. 03.022 .

Marani, A., R. Rigon, and A. Rinaldo (1991), A note on fractal channel networks, Water Resour. Res., 27(12), 3041-3049, doi:10.1029/ 91WR02077.

Maritan, A., A. Rinaldo, R. Rigon, A. Giacometti, and I. Rodríguez-Iturbe (1996), Scaling laws for river networks, Phys. Rev. E, 53(2), 1510-1515, doi:10.1103/PhysRevE.53.1510.

Montgomery, D., and W. Dietrich (1988), Where do channels begins?, Nature, 336, 232-234, doi:10.1038/336232a0.

Montgomery, D., and E. Foufoula-Georgiou (1993), Channel network source representation using digital elevation models, Water Resour. Res., 29(12), 3925-3934, doi:10.1029/93WR02463.

Moussa, R., and C. Bocquillon (1996), Criteria for the choice of floodrouting methods in natural channels, J. Hydrol., 186(1-4), 1-30, doi:10.1016/S0022-1694(96)03045-4.

Muzy, J., E. Bacry, and A. Arneodo (1994), The multifractal formalism revisited with wavelets, Int. J. Bifurcation Chaos Appl. Sci. Eng., 4, 245-302, doi:10.1142/S0218127494000204.

Nagatani, T. (1993a), Crossover scaling in Scheidegger's river network model, Phys. Rev. E, 47(6), 3896-3899, doi:10.1103/PhysRevE.47.3896.

Nagatani, T. (1993b), Multifractality of ow distribution in the river network model of Scheidegger, Phys. Rev. E, 47(1), 63-66, doi:10.1103/ PhysRevE.47.63.

O'Callaghan, J., and D. Mark (1984), The extraction of channel networks from digital elevation data, Comput. Vision Graphics Image Process., 28, 328-344.

Parisi, G., and U. Frisch (1985), On the singularity structure of fully developed turbulence, appendix to fully developed turbulence and intermittency by U. Frisch, in Proceedings of the International Summer School Physics Enrico Fermi, pp. 84-87, North Holland, Amsterdam.

Passalacqua, P., T. D. Trung, E. Foufoula-Georgiou, G. Sapiro, and W. E. Dietrich (2010), A geometric framework for channel network extraction from lidar: Nonlinear diffusion and geodesic paths, J. Geophys. Res., 115, F01002, doi:10.1029/2009JF001254.

Peckham, S., and V. Gupta (1999), A reformulation of Horton's laws for large river networks in terms of statistical self-similarity, Water Resour. Res., 35(9), 2763-2777, doi:10.1029/1999WR900154.

Pirotti, F., and P. Tarolli (2010), Suitability of lidar point density and derived landform curvature maps for channel network extraction, Hydrol. Processes, 24, 1187-1197, doi:10.1002/hyp.7582.
Rigon, R., I. Rodriguez-Iturbe, A. Maritan, A. Giacometti, D. G. Tarboton, and A. Rinaldo (1996), On Hack's law, Water Resour. Res., 32(11), 3367-3374, doi:10.1029/96WR02397.

Rigon, R., I. Rodriguez-Iturbe, and A. Rinaldo (1998), Feasible optimality implies Hack's law, Water Resour. Res., 34(11), 3181-3189, doi:10.1029/98WR02287.

Rinaldo, A., and I. Rodriguez-Iturbe (1998), Channel networks, Annu. Rev. Earth Planet. Sci., 26, 289-327, doi:10.1146/annurev.earth.26.1.289.

Rodríguez-Iturbe, I., and A. Rinaldo (Eds.) (1997), Fractal River Networks: Chance and Self-Organization, 1st ed., 540 pp., Cambridge Univ. Press, New York.

Rodríguez-Iturbe, I., E. J. Ijjasz-Vasquez, R. L. Bras, and D. G. Tarboton (1992), Power law distributions of discharge mass and energy in river basins, Water Resour. Res., 28(4), 1089-1093, doi:10.1029/ 91WR03033.

Scheidegger, A. E. (1967), A stochastic model for drainage patterns into an intramontane trench, Bull. Assoc. Sci. Hydrol., 12(1), 15-20, doi:10.1080/ 02626666709493507.

Schmidt, K.-H., and P. Ergenzinger (1992), Bedload entrainment, travel lengths, step lengths, rest periods studied with passive (iron, magnetic) and active (radio) tracer techniques, Earth Surf. Processes Landforms, 17(2), 147-165, doi:10.1002/esp.3290170204.

Shreve, R. (1966), Statistical laws of streams numbers, J. Geol., 74(1), 17-37, doi:10.1086/627137.

Shreve, R. (1967), Infinite topologically random channel networks, J. Geol., 75(2), 178-186, doi:10.1086/627245.

Strahler, A. N. (1952), Hypsometric (area-altitude curve) analysis of erosional topography, Geol. Soc. Am. Bull., 63, 1117-1141, doi:10.1130/ 0016-7606(1952)63[1117:HAAOET]2.0.CO;2.

Strahler, A. (1957), Quantitative analysis of watershed geomorphology, Eos Trans. $A G U, 74,913$.

Takayasu, H., I. Nishikawa, and H. Tasaki (1988), Power-law mass distribution of aggregation systems with injection, Phys. Rev. A, 37(8), 31103117, doi:10.1103/PhysRevA.37.3110.

Tarboton, D. G. (1992), A physical basis for drainage density, Geomorphology, 5, 59-76, doi:10.1016/0169-555X(92)90058-V.

Tarboton, D. G. (1996), Fractal river networks, Horton's laws and Tokunaga cyclicity, J. Hydrol., 187(1-2), 105-117, doi:10.1016/S0022-1694 (96)03089-2.

Tarboton, D. G., R. L. Bras, and I. Rodriguez-Iturbe (1991), On the extraction of channel networks from digital elevation data, Hydrol. Processes, 5(1), 81-100, doi:10.1002/hyp.3360050107.

Tarolli, P., and G. Dalla Fontana (2009), Hillslope-to-valley transition morphology: New opportunities from high resolution DTMs, Geomorphology, 113, 47-56, doi:10.1016/j.geomorph.2009.02.006.

Tucker, G., and K. Whipple (2002), Topographic outcomes predicted by stream erosion models: Sensitivity analysis and intermodel comparison, J. Geophys. Res., 107(B9), 2179, doi:10.1029/2001JB000162.

Valdés, J. B., Y. Fiallo, and I. Rodriguez-Iturbe (1979), A rainfall-runoff analysis of the geomorphologic IUH, Water Resour. Res., 15(6), 1421-1434, doi:10.1029/WR015i006p01421.

Veitzer, S. A., B. M. Troutman, and V. K. Gupta (2003), Power-law tail probabilities of drainage areas in river basins, Phys. Rev. E, 68, 016123, doi:10.1103/PhysRevE.68.016123.

P. Belmont, Department of Watershed Sciences, Utah State University, Logan, UT 84332, USA.

E. Foufoula-Georgiou, Department of Civil Engineering, University of Minnesota, Twin Cities, Minneapolis, MN 55414-2196, USA. (efi@umn.edu)

C. Gangodagamage, Earth and Environment Sciences Division, Los Alamos National Laboratory, Los Alamos, NM 87545, USA. 